\title{
Los medios como soportes de sistemas de representación: Implicaciones educativas
}

\section{Antonio Bautista}

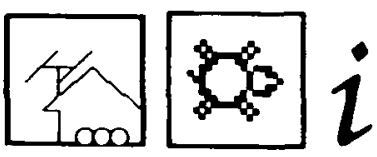

En esta investigación didáctica se experimenta con el aprendizaje $y$ uso de medios de creación y escritura audiovisual para establecer sus posibilidades educativas. El autor aboga por no encerrar los audiovisuales o los ordenadores en un «aula especial» sino integrarlos en el flujo educativo regular. La escritura audiovisual se presenta como una actividad marcadamente significativa para profesores y alumnos.

\section{REVISION, ENCUADRE Y PERSPECTIVA TEORICA}

\section{Antecedentes}

Los antecedentes de este estudio están situados dentro de dos «ejes de coordenadas»:

1) Los beneficios y ventajas que tienen los sistemas de representación para quienes poseen una amplia gama de ellos y, consecuentemente, las limitaciones y desventajas para quienes sólo dominan un reducido número de tales sistemas.

2) Los usos que se hace de los medios o recursos didácticos en la enseñanza. Existen diferentes tipos de usos, que se diferencian, entre otros aspectos, por la exigencia o no de la comprensión y utilización de códigos de representación.

Desarrollamos brevemente cada una de líneas que son antecedentes de este trabajo.

1) Los beneficios y ventajas de los sistemas de representación han sido puestos de manifiesto por diferentes autores en diferentes países y en diferentes momentos.

En el campo de la resolución, Mayer (1983) expresó que cuanto más sistemas de representación posea una persona más formas tendrá para representar los problemas que se le presenten y, consecuentemente, más fácil será para ese 
sujeto encontrar la solución, pues podrá observar más caminos que le lleven desde las condiciones del problema hasta la solución o soluciones.

Considerando los sistemas de representación como herramientas, autores como Vygotski (1979) y Giordan (1989) consideran que cuando una persona las utiliza en su relación con su contexto, modificándolo y siendo afectado por dicho cambio, internaliza su uso y características y, como consecuencia, modifica sus esquemas cognitivos, aumenta su conocimiento y puede, posteriormente, abordar con más calidad las dificultades y problemas que se le plantean en su entorno.

En un contexto clínico, Páez y cols. (1987) estudian las diferencias y convergencias entre las representaciones sociales y los esquemas cognitivos. Estos autores están observando, entre otros aspectos, que los sistemas de representación influyen en la percepción, inferencias, memoria, juicios y decisiones de las personas que los conocen.

Otra línea de investigación es la preocupada por definir los códigos y sistemas de representación que utiliza la mente para construir conocimientos o generar información nueva a partir de la que tiene almacenada (Clark, 1969; Simon, 1978; Denis, 1979; Sternberg, 1980; Rivière, 1986..). Entre otros aspectos, estos autores estudian los diferentes modelos de representación utilizados por los sujetos en los procesos de razonamiento cuando resuelven silogismos lineales o problemas de series de tres términos. Recientemente, Rivière (1986), teniendo presente la estructura y el contexto de los problemas (silogismos) presentados a los sujetos, ha presentado el razonamiento como un proceso de formulación y constrastación progresiva de hipótesis generadas por representaciones con diferentes niveles de profundidad. Concretamente dice: «Razonar consiste en abstraer representaciones suficientemente profundas de la información» (Rivière, 1986, p. 400).

Intentando situar las conclusiones de Rivière (1986) en un plano fenoménico, o aquel en el que un sujeto es capaz de ser consciente de cómo recibe y representa la información, hechos, objetos... de su contexto, consideramos que un sujeto comprende unos hechos, mensajes..., cuando les da significado, y esto sucede cuando los representa en los diferentes sistemas que posee. Cuantos más sistemas de representación posea más significado les dará, pues pasar un mensaje, concepto, objeto... a otro sistema necesita:

- hacer un análisis de los aspectos relevantes que constituyen ese objeto, hecho..., así como sus relaciones. De esta forma, puede:

- establecer la correspondencia entre cada uno de los elementos constitutivos del objeto y sus relaciones con los elementos y sus relaciones que constituyen el nuevo sistema donde se va a representar.

Este análisis es más profundo cuando el nuevo sistema de representación contiene unos elementos más alejados de la realidad o grado cero de representación, como ocurre con los lenguajes de ordenador, notas musicales... Estos son los sistemas de representación proposicionales y los procesos cognitivos que llevan a dar más significado a unos objetos o hechos del contexto mediante el paso de un sistema de representación a otro los consideramos como razonamientos en profundidad.

A su vez, cuando un sujeto ha representado un hecho, objeto, estado interno... en dos o más sistemas, entendemos que puede pensar ese hecho, objeto... de forma dialógica, estableciendo una conversación interna entre las diferentes represenaciones de la misma cosa. 
Estos estudios justifican que, inicialmente, consideremos a los sistemas de representación como:

- Sistemas que permiten realizar representaciones.

-Elementos relacionados que ayudan a representar problemas y encontrar más fácilmente sus soluciones.

-Entes beneficiosos, pues tales representaciones constituyen estructuras cognitivas que posibilitan enfrentarse eficientemente a fuentes de información ambiguas.

- Sistemas ventajosos, pues las representaciones hacen el mundo físico y social más predecible y significativo.

-Finalmente, los sistemas de representación son elementos básicos para que las personas que los poseen construyan un sistema cognitivo potente, bien elaborado y, por tanto, con posibilidad de razonar, relacionarse con el contexto social, físico y cultural en el que vive.

2) El segundo eje de coordenadas que sitúa este estudio está vinculado a los diferentes tipos de usos que se hacen de los medios en la enseñanza.

Esta elección hace referencia a los distintos procesos seguidos por profesores y alumnos, así como a las distintas dimensiones y elementos tenidos en cuenta, hasta llegar a una decisión sobre qué medios se van a utilizar y qué usos se van a hacer para realizar unas tareas que conlleven un aprendizaje y un desarrollo personal.

Sin la evidencia de los datos de una investigación, pero con los argumentos expuestos en líneas anteriores, entendemos que la calidad, bondad o méritos de una modalidad de uso está en función del nivel de exigencia que se hace al alumno en cuanto a la comprensión y utilización de sistemas de representación. Esto nos lleva a realizar unos planteamientos de usos de medios de la enseñanza que van más lejos de los propuestos en las décadas precedentes.

\section{Tipos de usos de medios}

En los años 50 y 60, autores como Lefranc (1969) y Goodwin (1972) consideraban los recursos audiovisuales como elementos válidos para despertar la atención de los alumnos, para comunicar y repetir informaciones, para que retengan hechos, para fomentar actitudes y valores positivos, y lo que se consideraba más importante, para acelerar el proceso de aprendizaje. Puede observarse que el fundamento de estas primeras aplicaciones de los medios se encuentra en las teorías psicológicas que explican el aprendizaje.

En la década de los 70 prioritariamente, los medios son considerados como aquellos recursos basados en el desarrollo de unos materiales que permiten a los alumnos conseguir unos objetivos operativos que han sido definidos previamente (Tosti y Ball, 1969; Briggs, Campeau, Gagné, May, 1973; Hallas, 1978...).

Consideramos que estos usos de los medios que aún se realizan en diferentes ámbitos y países no exigen un conocimiento y utilización de los sistemas de representación, con lo que evita y priva a los alumnos de beneficios y ventajas señalados anteriormente, pues este uso se limita a presentar informaciones, temas, mensajes, etc., soportados en programas, vídeos didácticos,..

Como ya hemos puesto de manifiesto en otro trabajo (Bautista, 1989), para evitar estas limitaciones, es necesario hacer un uso comprensivo o situacional y crítico de los medios.

Así, en los usos comprensivos/situacionales, el profesor, partiendo del cono- 
cimiento del contexto del aula, tendrá más posibilidad de diseñar tareas comprensivas y llenas de significado para los alumnos. Tareas coherentes que pueden estar inscritas dentro de situaciones problemáticas o de proyectos de trabajos. Dentro de este uso, los equipos de vídeo, ordenador..., además de ser elementos de ayuda para ejecutar los planes elaborados por los alumnos con el fin de resolver esos problemas o desarrollar tales proyectos, proporcionan y hacen posible utilizar sistemas de representación.

\section{Selección de usos de medios}

A falta de una investigación seria que confirme o refute estas sospechas, parece evidente que los beneficios de los medios residen, en buena medida, en el uso que se haga de ellos. Esta importancia de los modos de utilización de los medios lleva a cuestionarnos por la forma como se seleccionan esos recursos en la enseñanza.

Los primeros planteamientos orientaron la selección de medios con unos criterios de disponibilidad, economía y por los posibles procesos (motivación, atención, comunicación...) que quieren poner en funcionamiento (Lefranc, 1969; Goodwin, 1972; Norbis, 1972...).

Unas perspectivas más sistemáticas orientan la selección de medios desde unos procedimientos o algoritmos que tienen presentes los objetivos definidos en términos de conducta, los estímulos, las posibles respuestas de los alumnos, la gestión planificada por el profesor (Tosti y Ball, 1969; Brigg y cols., 1978; Hallas, 1978; Romiszowski, 1988; ...). Muchos de ellos, según Gimeno (1981), olvidan que el contexto es quien da el valor a los medios y que, dependiendo de estas situaciones de enseñanza, un mismo medio puede tener una u otra función. Así, otras funciones de los medios además de la de comunicar informaciones, son el uso que hacen los alumnos para resolver problemas, desarrollar proyectos de trabajo...

Estos planteamientos en la selección de medios en la enseñanza son limitados, pues no tienen presente el contexto físico, social y cultural donde se usa el medio, ni los efectos secundarios o no previstos en la enseñanza interactiva, ni las ventajas que posiblemente tienen los sistemas de representación. Es preciso, pues, plantear un modelo de selección de usos de medios que contemple estas dimensiones de la enseñanza y, consecuentemente, evita las posibles dificultades y limitaciones que puede ocasionar una mala utilización de los mismos.

\section{b) Estado actual de la cuestión. Se expresa en estos puntos:}

- Estudios sobre TV.

Para estudiar los beneficios que tenía el uso de la TV en la enseñanza, Salomon y Cohen (1977) distinguieron entre utilizar los programas que se emitían por TV (uso del monitor o tecnología de la TV) y el uso de los sistemas simbólicos del lenguaje de la imagen dinámica o móvil.

Desde esta última perspectiva, los estudios de Olson $(1974,1977)$, Schramm (1977) y Salomon $(1979,1984)$ han demostrado que las características simbólicas de los medios pueden tener un efecto positivo en el desarrollo cognitivo y que estos efectos no tiene lugar, necesariamente, como resultado de una exposición al medio que sea aleatoria y sin planificar. También se desprende de sus investigaciones que los efectos cognitivos dependen de una variedad de factores 
como son el esfuerzo realizado, niveles de profundidad del procesamiento de la información y de las aptitudes de los alumnos.

Recientemente, y ante las aportaciones de los estudios de la Psicología, senaladas anteriormente (Rivière, 1986) como que las operaciones cognitivas básicas se apoyan en estructuras proposicionales, Clark y Salomon (1986) entienden que los medios más que elementos generadores de habilidades cognitivas son instrumentos que dificultan o facilitan el paso de la información a sistemas proposicionales.

- Investigaciones sobre lenguajes de programación y desarrollo cognitivo (preferentemente con Logo). Es otra línea actual de investigación sobre los beneficios y ventajas de los medios. Autores como Kurland y Pea (1984), Hawkins, Kurland y Pea (1985) y la revisión realizada por Ginter y Willianson (1985) han puesto de manifiesto que no hay unos resultados concluyentes y que el lenguaje de programación LOGO no puede considerarse una panacea como pretendía Papert (1982).

Sin duda, los lenguajes de ordenador, como sistemas de representación, pueden tener una gran utilidad, pero ésta dependerá de cómo se usen e integren en las tareas que tienen lugar dentro del aula.

Hemos observado que no hay unos resultados concluyentes en el estado actual del tema y, sobre todo, que la mayoría de estas investigaciones se han realizado en unas condiciones «artificiales» o alejadas del contexto natural del aula y, por tanto, carecen de validez ecológica. Así los diversos estudios sobre Logo:

- Los alumnos realizan unas tareas que están alejadas de los contenidos y contexto del aula, como son realizar rompecabezas, la torre de Hanoi, pruebas de permutaciones...

- Se han desarrollado en períodos cortos de tiempo, con lo que no controlan el efecto «novedad» del medio y no da tiempo para que los alumnos asimilen los beneficios de estos sistemas de representación. Por ejemplo, Clements y $\mathrm{Gu}$ Ilo (1985) desarrollaron en su estudio dos sesiones de 40 minutos durante 12 semanas. Gorman y Bourne (1983) y Kurland y Pea (1985) trabajaban utilizando Logo con sus alumnos durante una media de 30 horas en total.

- Los efectos de los sistemas simbólicos (lenguajes de programación y de la imagen en movimiento) se han valorado, principalmente, sobre el rendimiento académico (nivel de conocimientos) y sobre la capacidad de planificar.

- Sólo Kurland y Pea (1985), sin llegar a llevarlo a efecto, pusieron de manifiesto la necesidad de estudiar el uso hecho de los medios por el profesor y los alumnos.

Ante este estado del tema de estudio, para nuestros propósitos, es preciso estudiar qué beneficios y ventajas proporcionan a los alumnos un uso comprensivo y crítico de los medios, según se definió líneas atrás, que exija conocer y utilizar dos sistemas de representación: los lenguajes de programación y el lenguaje de la imagen dinámica. Ante estos resultados, podremos valorar desde un punto de vista del control y de las diferencias sociales los principios incluidos en los diseños curriculares que orientan la selección y los usos que se deben hacer de los medios.

Finalmente, hemos de indicar que en nuestro país hay tres grupos que trabajan en este campo, si bien con unos propósitos diferentes al nuestro. El grupo dirigido Benedito (1988) en la Universida Central de Barcelona y el de Del Val (1988) en la Universidad Autónoma de Madrid sobre Logo y desarrollo intelec- 
tual, y los estudios que realiza San Martín (1986) en la Universidad de Valencia sobre el pensamiento del profesor y el uso de los medios audiovisuales.

\section{PROPOSITOS DEL ESTUDIO}

Ante el anterior marco de análisis, el propósito de este trabajo es estudiar:

1) Qué beneficios y ventajas suponen para los alumnos la asunción y uso de dos sistemas de representación, el lenguaje de programación Logo y el lenguaje cinematográfico. Es decir, comprobar en un contexto de aula y durante un tiempo prolongado, tres cursos escolares, si se producen los beneficios que se anuncian desde diferentes marcos teóricos y que no coinciden con las experiencias realizadas en contextos de «laboratorio».

Concretamente, pretendemos comprobar si la comprensión y uso de esos esquemas permite a los sujetos:

- Expresar ideas o abstracciones de diferentes formas.

- Comunicar aspectos concretos de la realidad (hechos, objetos...) de diferentes formas.

- Comprender diferentes mensajes e informaciones proporcionados por las nuevas tecnologías (imagen dinámica y ordenadores) y que están soportados en diferentes códigos de representación.

- Abordar de diferentes formas los problemas y dificultades de naturaleza diversa (del hogar, calle, disciplinas académicas...).

- Realizar razonamientos diferentes en profundidad y en paralelo.

- Generar experiencias vitales que les permita mantener unas relaciones valiosas a nivel familiar, social...

-Otros aspectos relevantes de significado que surjan en los procesos de enseñanza y de aprendizaje ocurridos en el aula.

2) Si estos beneficios y ventajas están vinculados al uso que se hace de los medios. Concretamente, qué usos de los recursos son los que llevan a asumir y usar con más coherencia y calidad los sistemas de representación.

3) Consecuentemente, las deducciones o derivaciones que surgen de comprobar la validez del siguiente argumento:

- Si ciertos beneficios de los medios y materiales están condicionados por los usos que se hacen de ellos (favorecer o evitar el aprendizaje y uso de sistemas de representación), y

-si los usos están determinados/condicionados por el control técnico o de las prescripciones administrativas realizadas en los diseños curriculares, entonces

- los beneficios culturales y cognitivos de los medios y, consecuentemente, las diferencias sociales, están condicionados por el control técnico que se hace de los usos a través de los materiales y otros códigos del currículum.

\section{FUNDAMENTOS METODOLOGICOS}

Para estudiar los efectos de los diferentes sistemas de representación en los alumnos y los procesos internos del pensamiento del profesor cuando selecciona esos códigos de representación y el uso de medios, aconsejan que nos situemos dentro de los paradigmas científicos interpretativos y, por tanto, utilicemos 
un modelo de investigación cualitativo basado en el estudio de casos. Los datos e informaciones los obtendremos, pues, mediante entrevistas y observación directa y de grabaciones en vídeo de la práctica. La subjetividad de las interpretaciones será controlada usando procesos de triangulación.

Una de las características relevantes de los modelos cualitativos es realizar la obtención y el análisis de datos e informaciones de forma simultánea y coordinada a lo largo de toda la investigación. Esto significa que, aunque el investigador vaya a su campo de estudio (aula en nuestro caso) con algunas unidades de análisis y estrategias de recogida de datos definidas previamente, utilizará las informaciones que vaya recogiendo para volver a especificar los elementos de observación posteriores.

De los anteriores procesos integrados de observación y análisis surgen una serie de categorías que caracterizan y explican los fenómenos complejos que son objeto de estudio.

Otro de los aspectos básicos de los métodos de investigación cualitativos hace referencia a la interpretación e integración de las categorías relevantes que han emergido de ese estudio con las de otras investigaciones relacionadas para poner de manifiesto cómo aquél se encuadra en marcos teóricos más amplios que, a su vez, darán más significado a los problemas y campos objeto de solución y conocimiento.

\section{SECUENCIA Y MODO DE APLICACION}

Para conseguir los propósitos señalados y evitar las deficiencias y limitaciones de los estudios reseñados en el apartado anterior, este trabajo tiene dos características:

- Integración del uso de los medios y de los sistemas de representación que conllevan ese uso, en un contexto de aula, es decir, en las tareas a través de las cuales se asumen los contenidos o elementos de la cultura.

- Observar los procesos seguidos por profesores y alumnos desde cerca y durante un tiempo prolongado.

Con estas intenciones:

Seleccionamos a dos grupos de alumnos (casos sobre los que haremos el seguimiento de los procesos) que estaban al principio, año escolar 1990/1991, en sexto curso de EGB. Ambos grupos pertenecen a dos colegios públicos.

Seguimos el siguiente proceso en el tiempo:

\section{Primer año: Curso escolar 1990/1991}

Los dos grupos de EGB son de sexto curso. Como en Ciclo Superior normalmente hay cuatro profesores que imparten clase a un grupo, participan ocho docentes en total.

Durante este primer año se desarrollaron las siguientes fases:

a) Concreción del plan de trabajo en cada centro con los profesores respectivos.

b) Proporcionar los sistemas de representación (lenguaje cinematográfico y lenguaje de programación LOGO) a los alumnos de ambos grupos.

Durante este año escolar hubo uno o dos observadores externos por aula 
que dedicaron al menos 6 horas de observación a cada uno de los grupos.

Consideramos que este tiempo semanal durante casi tres cursos escolares es básico y suficiente para poder percibir cómo los alumnos asumen los sistemas de representación y van construyendo significados, y respecto a los profesores cómo seleccionan y usan los medios y los códigos de representación.

\section{Segundo y tercer año: Cursos escolares 1991/1992 y 1992/1993}

Durante estos años, los dos grupos de alumnos seleccionados como «casos» para el estudio son los mismos del año anterior que estarán en séptimo de EGB en el curso académico 91/92, y en octavo durante el 92/93.

Los profesores que tuvieron los alumnos en sexto curso, seguirán siendo los mismos en séptimo y octavo con el fin de mantener la coherencia y línea de trabajo.

Los observadores externos, cada uno en su grupo, continuarán observando los procesos seguidos por profesores y alumnos en el aula, y realizando entrevistas de seguimiento sobre las ventajas y beneficios proporcionados por los sistemas de representación con el fin de elaborar constructos que ayuden a comprender y explicar estos beneficios y los usos y selección de medios realizados por el profesor.

\section{IMPLICACIONES PARA EL TRABAJO EN EL AULA}

\section{a) Para llegar a los objetivos propuestos, se realizan las siguientes acciones de planificación al principio de cada uno de estos dos cursos académicos:}

- Seleccionar contenidos. Cada profesor elegirá el grupo de contenidos de su área de conocimiento que desee se trabaje de forma interdisciplinar a través del desarrollo de proyectos de trabajo o de situaciones problemáticas.

-Elaborar proyectos y situaciones problemáticas vinculadas con sus entornos primarios, que durante su desarrollo tengan que utilizar los diferentes sistemas de representación que aprendieron el año anterior. A su vez, durante esos procesos de trabajo, los alumnos tendrán que acceder a fuentes de información, rastrear datos, proponer y comprobar hipótesis, y, consecuentemente, asumirán los diferentes contenidos o elementos de la cultura y usarán los medios como soporte de sistemas de representación de esas situaciones, acontecimientos, principios científicos...

Tales proyectos surgen del grupo y se desarrollan en el aula y fuera de ella en tiempo extraescolar. Los observadores y profesores mantendrán un seguimiento próximo de tales trabajos. Paralelamente, cada profesor irá trabajando el resto de los contenidos de sus asignaturas respectivas con otras tareas y ritmos que consideren pertinentes.

Un ejemplo de proyecto que permite a los alumnos de un aula trabajar y entender el contexto cultural, social, arquitectónico, político, lingüístico, etc. de Francia a finales del siglo XVIII y principios del XIX, es el siguiente:

«Hacer un vídeo de duración moderada donde se exprese qué posibles acontecimientos, culturales, políticos... habrían cambiado si Napoleón Bonaparte hubiese sido soldado durante toda su vida militar.» 
Si en ese centro escolar no dispusiesen de cámara de vídeo, el proyecto se podría centrar en la elaboración de un cómic.

Una característica de las aulas donde se desarrolla un proyecto de ese tipo es la pluralidad de tareas que paralela y simultáneamente se realizan. Así, mientras que un grupo está rastreando una información en una enciclopedia para completar el guión literario, otro grupo está preparado unos decorados, otro está grabando un detalle con el «macro» de la cámara...

\section{b) Abajo los centros de recursos; los medios, ... al aula.}

Otra peculiaridad de esa forma de trabajar es que los medios estén en las aulas. Los usos clásicos o transmisores de esos equipos tecnológicos conllevan una organización de los mismos en aulas y centros de recursos al frente de los cuales, y dependiendo de la envergadura de los mismos, habrá un experto o equipo de expertos que, ante las demandas realizadas por los profesores sobre relaciones de objetivos terminales y mensurables a conseguir, recomendarán unos medios frente a otros.

Esta estructura organizativa, a su vez, condiciona profundamente la selección de medios que realice el propio profesor, ya no solamente durante situaciones preactivas, sino interactivas. Pues cuando en la interacción surja un problema de comprensión, una alteración del orden básico necesario en los espacios educativos, o cualquier otra dificultad no prevista, el profesor no podrá detener el curso de la acción para ir a consultar al experto y que éste le recomiende unos medios y unos usos para dar respuesta a tales dificultades emergentes. Evidentemente, el profesor es el único que conoce esas situaciones y, consecuentemente, el único que podrá hacer una elección adecuada de usos de medios o de algún material para darles unas soluciones satisfactorias, significativas y coherentes. Así pues, la organización de los medios en centros de recursos evita la disponibilidad de los medios que tienen los profesores en el proceso, pues seleccionan entre los recursos disponibles en los espacios y en el momento que aparecen las situaciones conflictivas.

Estos argumentos nos llevaron a proponer y materializar el siguiente eslogan: «Abajo los centros de recursos; los medios, ... al aula». Consecuentemente, los diferentes equipos se distribuyen por las clases para que los alumnos se dirijan a ellos cuando tengan que comprobar alguna hipótesis, acceder a alguna información... Es una organización de medios que respeta el plan de actuación y no rompe la dinámica generada en el aula.

\section{c) ¿Cómo trabaja cada día cada uno de los observadores?}

- Para ir construyendo las categorías conceptuales que ayuden a dar respuesta a los interrogantes de este estudio, preocupará observar todo lo que ocurre en el aula y, sobre todo, aquellos aspectos que son relevantes y esenciales para comprender la interacción y los significados generados. De forma especial observará cómo usa el profesor los medios y qué sistemas de representación «obliga» o «evita» que utilicen los alumnos. Respecto a los alumnos, procurará observar los beneficios que proporcionan los sistemas de representación cuando están desarrollando tareas planificadas por el profesor. Los observaremos por los procesos y estrategias de resolución de problemas que siguen, por la influencia de los códigos de representación en el aumento de la comprensión y del significado 
que generan, por las formas y recursos en su comunicación, y por todos los aspectos no previstos que se pongan de manifiesto en el proceso de enseñanza del aula.

Además de la observación directa, el observador utilizará grabaciones en vídeo para, en los visionados posteriores, obtener datos de la interacción simbólica desarrollada en los espacios de trabajo.

- El observador hará entrevistas al profesor y alumnos después de la clase para obtener informaciones sobre cómo han asumido estos últimos las tareas, su nivel de entusiasmo o apatía, el nivel de comprensión de los contenidos..., y para constatar los beneficios de los sistemas de representación al comprobar relaciones entre las manifestaciones retrospectivas que proporcionan o proporcionaron sobre los usos de los medios y los síntomas objetivos (observación) de haberlos utilizado.

Estas entrevistas, pues, servirán para ir elaborando continuamente categorías de observación y constructos que ayuden a dar respuesta a los interrogantes planteados en este estudio. Las entrevistas serán grabadas en audiocasete.

- Normalmente, todos los observadores mantenemos una reunión al mes, para deliberar sobre las posibles categorías de observación que se van generando en los grupos, sobre los beneficios o limitaciones que van emergiendo de los sistemas de representación utilizados, y sobre la relación que ambos tienen con el uso que se hace de los medios en el aula.

\section{d) ¿Qué efectos tienen en los alumnos esas formas de trabajar?}

A falta de analizar sistemáticamente todos los datos obtenidos durante el año académico 1990/1991 para generar unas categorías que los expliquen, queremos expresar algunas manifestaciones de alumnos, que consideramos relevantes para entender el significado que confieren a las situaciones educativas originadas. Se pueden resumir en las siguientes:

«... los debates que se crearon cuando teniamos que utilizar la cámara de vídeo para expresar sentimientos, así como las manifestaciones de las vivencias y del mundo interior de los compañeros, ban sido la base de algunas de las añoranzas del futuro...»

«... si de mayores pensamos en la escuela, seguro que recordaremos estos trabajos en grupo que han generado unas experiencias que son vitales para conocernos y relevantes para entender mejor el mundo donde vivimos..."

Terminamos, pues cualquier comentario a tales manifestaciones consideramos que sería vano y superfluo. 


\section{Referencias}

Bautista, A. (1989). «Los usos de los medios desde las teorías del currículum. Comunicación Lenguaje y Educación, 3.4, pp. 39-52.

Benedito, V. (1988). «Introducción de la informática en la enseñanza». En VV. AA. Cuestiones de didáctica. Ceac. Barcelona.

BRIGGS, L.J. y otros. (1973). Los medios de la instrucción. Guadalupe. Buenos Aires.

Clark, H.H. (1969). "Linguistic processes in deductive reasoning». Psycological Review. 76, pp. 378-404

Clatk, R.W. y G. Salomon. (1986). «Media in Teaching». En WITTROCK, M.C. (Ed.) Handbook of Research in Teaching. MacMillan Publising Co. New York. pp. 464-478.

Clemfnts, D.H. y GuLno, D.F. (1984). «Effects of the computer programming on the young children's cognition». Joumal of Educational Psychology. 76,6; pp. 1051-1058.

Delval, J. y cols. (1988). «Logo y desarrollo intelectual». Actas del Simposio Internacional de Educación e Informática. ICE UAM.

Denis, M. (1979). Les images mentales. PUf. París. (Traducido en editorial Siglo XXI. Madrid. 1984).

Gimeno, J. (1981). Teoria de la enseñanza y desarrollo del curriculum. Anaya. Madrid.

Ginther, D.W. y Willianson, J.D. (1985). «Learning Logo: What is Really Learned». Computers in the School. 2, 2/3; pp. 73-78.

Giondan, A., (1989). «Representaciones sobre la utilización didáctica de las representaciones». Enseñanza de las Ciencias. 7 (1), pp. 53-62.

Goodwin, A.B. (1972). Manual de medios audiovisuales para la Educación General Básica. Paraninfo. Madrid.

Hallas, J. (1978). «Audio-visual techniques for industry». Development and Transfer of Tecbnology. 88, pp. $31-56$.

Hawkins, J. Kurland, D.M y PeA, R.D. (1985), «Logo and the development of thinking skills». En Chen, M. y Paisley, W. (eds.). Children and microcomputers: research on the newest medium. Beverly Hills. California.

KERR, S.T. (1981). «How teachers desing their materials: implications for instructional desing». Instructional Science, 10 , pp. 363-378.

KuRland, D.M. y PEA, R. (1984). «On the cognitiva effects of learning computer programming». New ideas in psychology. 2,2, pp. 137-168.

Lefranc, R. y cols. (1979). Las técnicas audiovisuales al servicio de la enseñanza. El Ateneo. Buenos Aires.

MaYer, R.W. (1983). Thinking, Problem Solving, Cognition. Freeman and Company. New York.

OLson, D. (1977). "Oral and written communication and the cognitive processing of children». Joumal of Communication, 27, pp. 10-26.

Páez, D. y cols. (1987). Pensamiento, individuo y sociedad. Cognición y representación social. Fundamentos. Madrid.

Papert, S. (1982). Desafío a la mente. Galápago. Buenos Aires.

RIvière, S. (1982). Razonamiento y representación. Ed. Siglo xxı. Madrid.

Romiszowski, A. (1988). Selection and use of Instructional Media. Kegan Page. Londres.

SALOMON, G. (1979). Interaction of media, cognition and leaming. Jossey-Bass. San Francisco.

SALOMON, G. (1979). «Media and simbol systems as related to cognition and learning». Joumal of Educational Psychology. 71, 2; pp. 131-148.

SALOMON, G. y GARDNER, H. (1984). The computer as educator: Lessons fron television research. Harvard University. MA.

SAn Martin, A. (1986). Los recursos audiovisuales en el pensamiento pedagógico del profesor. Nau Libres. Valencia.

Schramm, W. (1977). Bag media: Little media. Beverly Hills. CA.

SIMON, H.A. (1978). «On the forms of mental representation». En SAVAGE, C.W. (Compilador). Perception and cognition issues in the foundation of psychology. University of Minnessota Press. Minneapolis.

STERNBERG, R.J. (1989). «Representation and process in linear syllogistic reasoning». Joumal of Experimental Psychology. 109, pp. 119-159.

Tosti, D. y J. Ball. (1969). «A behevioral approach to instruction, desing and media selection». Audio Visual Communication Review, 17, pp. 5-25.

VyGoTsKı, L.S. (1979). El desarrollo de los procesos psicológicos superiores. Crítica. Barcelona. 


\section{Los medios como soportes de sistemas de representación: Implicaciones educativas.}

\section{Antonio Bautista. \\ CL\&E, 1992, 14, pp. 77-88}

Resumen: El propósito principal de este artículo es adelantar un breve informe de una investigación en curso donde se analiza el valor que tiene la organización de los medios en los ámbitos escolares y los usos que se hacen de ellos cuando es preciso conocer y utilizar sistemas de representación (lenguaje cinematográfico, de ordenador,..). Un aspecto importante que se está poniendo de manifiesto es que son los contextos organizativos y sociales y los usos que se hacen de los recursos y de los materiales quienes determinan, en gran medida, el efecto que tienen sobre el pensamiento de quienes los utilizan.

Datos sobre el autor: Antonio Bautista Garcla es profesor en el Departamento de Didáctica y Organización Escolar en la Facultad de Pedagogía de la Universidad Complutense de Madrid. Lleva trabajando varios años sobre temas del Curriculum y del uso, selección, organización y evaluación de recursos y materiales en ámbitos educativos.

Dirección: Universidad Complutense. Facultad de Pedagogía. Departamento de Didáctica y Organización Escolar. Edificio de la Almudena. Avda. Juan XXIII, s/n. Campus de la Complutense. Madrid.

(C) De todos los artículos deberá solicitarse por escrito autorización de CL\&E y de los autores para el uso en forma de facsímil, fotocopia o cualquier otro medio de reproducción impresa. CL\&E se reserva el derecho de interponer las acciones legales necesarias en aquellos casos en que se contravenga la ley de derechos de autor. 